EPJ Web of Conferences 41, 05026 (2013)

DOI: $10.1051 /$ epjconf/20134105026

(C) Owned by the authors, published by EDP Sciences, 2013

\title{
Ultrafast spectroscopy of linear carbon chains: the case of dinaphthylpolyynes
}

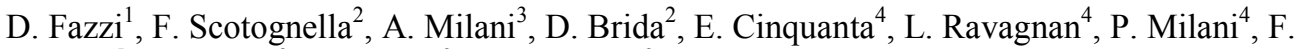 \\ Cataldo $^{5}$, M. Negro ${ }^{2}$, S. Stagira ${ }^{2}$ and C. Vozzi $^{2 \star}$ \\ ${ }^{1}$ Center for Nano Science and Technology CNST@Polimi, Istituto Italiano di Tecnologia, via \\ Pascoli 70/3, 20133 Milano, Italy \\ ${ }^{2}$ CNR-IFN and Dipartimento di Fisica, Politecnico di Milano, Piazza L. da Vinci 32, 20133 Milano, \\ Italy \\ ${ }^{3}$ Dipartimento di Chimica, Materiali e Ing. Chimica “G. Natta”, Politecnico di Milano, Piazza L. da \\ Vinci 32, 20133 Milano, Italy \\ ${ }^{4}$ CIMAINA and Dipartimento di Fisica, Universita` di Milano, via Celoria 16, 20133 Milano, Italy \\ ${ }^{5}$ Actinium Chemical Research s.r.l., Via Casilina 1626/A, 00133 Roma, Italy \\ e-mail: caterina.vozzi@polimi.it
}

\begin{abstract}
The dynamics of excited states in a special class of linear carbon chains, e.g. dinaphthyl polyynes, has been investigated by ultrafast transient absorption spectroscopy and DFT//TDDFT calculations. The ultrafast formation of a narrow photoinduced absorption band has been observed and assigned to an inter-system crossing event bringing to the formation of triplet excited states.
\end{abstract}

\section{Introduction}

Polyynes are a class of linear carbon chains consisting of alternating single and triple carbon bonds $(\mathrm{C}-\mathrm{CC} / \equiv \mathrm{C})$. Among the wide variety of carbon based materials, these systems have recently been attracting a great interest because of their unique physical and chemical properties [1,2]. Indeed, sp-hybridized carbon chains (spCCs) are found in interstellar space, biological systems and are extensively used as building blocks in supramolecular chemistry. Recent experimental and theoretical predictions, describing both linear and nonlinear optical responses of polyynes, support the fact that spCCs show the behaviour expected for true one-dimensional conjugated systems, resulting in large second order hyper-polarizability, paving the way for possible nonlinear optical applications. In addition the stiffness of the triple/single carbon bond chain is particularly interesting for nanotech applications and is higher than in carbon nanotubes [3].

Here we report a photo-physical study on a class of polyynes, namely $\alpha, \omega$-dinaphthylpolyynes Ar-C2n-Ar (with Ar naphthyl group and $\mathrm{n}$ the number of triple bonds), by carrying out a joint experimental and theoretical investigation. Ultrafast transient absorption spectroscopy and first principles quantum chemical investigations (DFT and TD-DFT methods) are here applied to study the singlet and triplet excited states energies, dynamics and internal conversions processes.

This is an Open Access article distributed under the terms of the Creative Commons Attribution License 2.0, which permits unrestricted use, distribution, and reproduction in any medium, provided the original work is properly cited. 


\section{Results and discussion}

Ar-C2n-Ar are synthesized by reacting copper(I)ethynylnaphthalide with diiodoacetylene under the Cadiot-Chodkiewicz reaction conditions [4]. The batch obtained consists in a decaline solution with dinaphthylpolyynes having different chain lengths. Among these, the most concentrated species are characterized by $n=2,3,4$ triple bonds. The absorption spectra of these species, acquired by liquid chromatography (HPLC) [3], are shown in Figure 1(a).

(a)

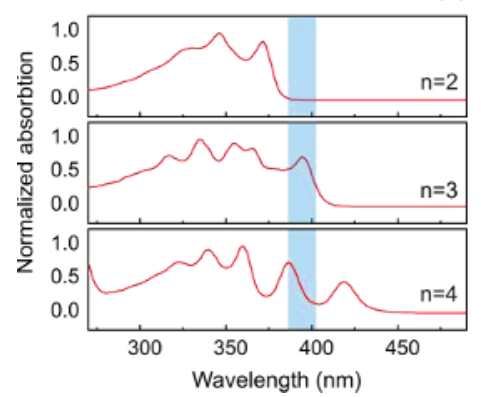

(b)

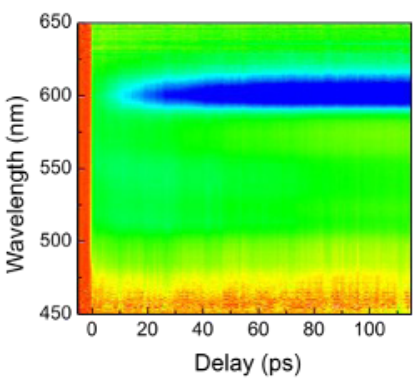

(c)

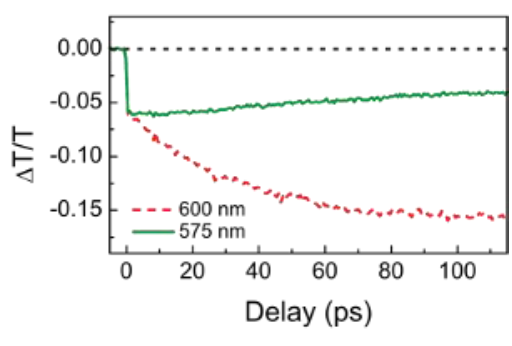

Fig. 1. (a) Absorption spectra of polyynes with different chain length, the shadow indicates the spectral region corresponding to the excitation. (b) Contour plot of the pump probe measurement. (c) Transient absorption dynamics for two probe wavelengths.

The time resolved experiments were performed by exciting the sample with a 150 -fs pulse centred at $390 \mathrm{~nm}$ derived as the second harmonic of a regenerative amplified Ti:Sapphire laser. This pump beam was resonant only with the polyynes with $n \geq 3$. The probe was obtained by white light generation in a 3-mm thick sapphire plate and then coupled to a fast CCD array working at the full repetition rate of $1 \mathrm{kHz}$. The transient absorption spectrum as a function of the probe wavelength is then acquired by modulating the pump beam with a mechanical chopper. Figure 1(b) shows the 2D map of the pump-probe signal. Over the whole spectral bandwidth it is possible to observe a broad photo-induced absorption that appears instantaneously upon impulsive photo-excitation. This feature can be assigned to optical transitions from the excited singlet state to higher molecular singlet levels. Furthermore, a clear photo-induced absorption signal forms at $600 \mathrm{~nm}$ with a time constant of $31 \mathrm{ps}$ and a narrow-band spectral shape. This component can be assigned to the formation of a triplet state. In Figure 1(c) are shown two cuts of the 2D map at selected wavelength that track the internal conversion dynamics. Another set of measurements performed by exciting the sample at $420-460 \mathrm{~nm}$ did not show any signature of the triplet state, thus ruling out its formation on the picosecond time scale for polyynes with $\mathrm{n} \geq 4$ that can be excited at this wavelength. Our measurements highlight that the fast internal conversion mechanism occurs only for short polyyne length.

DFT (B3LYP/cc-pVTZ) and TDDFT calculations, carried out on the Ar-C2n-Ar (n=2-4) species, unveil the ground and excited states properties of these mono-dimensional carbon based systems. The ground to excited state transitions (e.g. S0 $\rightarrow$ Sn) have been calculated for each chain length and the character of the first absorption band $(\mathrm{S} 0 \rightarrow \mathrm{S} 1)$ has been assigned, in terms of molecular orbitals, to the $\mathrm{H} \rightarrow \mathrm{L}$ and $\mathrm{H}-1 \rightarrow \mathrm{L}+1$ transitions, as reported in Figure 2(a). The Franck-Condon factors (e.g. vibronic activity) have been evaluated (TD-B3LYP/cc-pVTZ) by calculating both the molecular structures and force fields in the low lying singlet excited states. The normal modes governing the vibronic progression observed in the absorption spectra have been identified and assigned to the in phase stretching and shrinking variations of the $\mathrm{C} \equiv \mathrm{C} / \mathrm{C}-\mathrm{C}$ units of the carbon chain. 

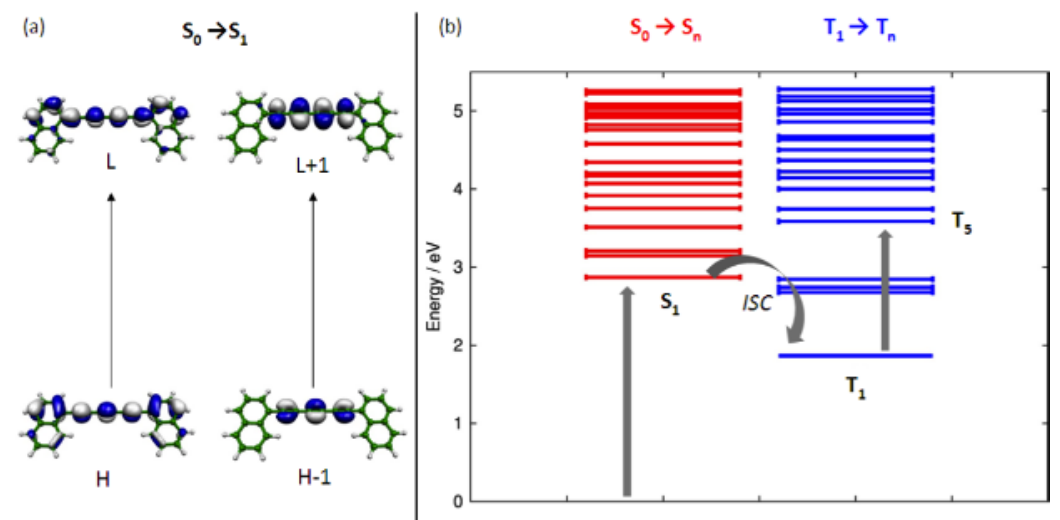

Fig. 2. TD-B3LYP/cc-pVTZ calculated molecular orbital mainly involved in the $S_{0} \rightarrow S_{1}$ transition. (b) TD(U)B3LYP/cc-pVTZ calculated singlet and triplet excited state energies. In figure is also reported the $\mathrm{T}_{1} \rightarrow \mathrm{T}_{5}$ dipole allowed transition for the $\mathrm{Ar}-\mathrm{C}_{6}-\mathrm{Ar}$.

To get insights in the pump probe maps and dynamics (Figure $1(b, c)$ ) the first triplet $\left(T_{1}\right)$ excited state has been optimized at the UB3LYP/cc-pVTZ level and the $T_{1} \rightarrow T_{n}$ excited state transitions have been evaluated at the TD-UB3LYP/cc-pVTZ level as well. For both $n=3,4$ polyynes we found an optically allowed $\mathrm{T}_{1} \rightarrow \mathrm{T}_{5}$ transition at $1.87-1.81 \mathrm{eV}$ (e.g. 660-683 nm) with high oscillator strengths $(\mathrm{f}=1.3-1.4)$ respectively (see Figure $2 \mathrm{~b}$ ). The calculated triplet-to-triplet transitions are in good agreement with the observed photo-induced absorption signal at $600 \mathrm{~nm}$ and the corresponding dynamics in Figure 1b,c. TDDFT calculations support also the fact that the 600nm photo-induced absorption signal corresponds to a very narrow-band. From the computational point of view, the explanation of the ultrafast internal conversion process that takes place only for $n=3$ is still elusive.

In conclusion, we observed a fast singlet to triplet internal conversion mechanism revealed by the ultrafast photo- induced absorption narrow band at $600 \mathrm{~nm}$. Excited states TDDFT calculations assigned the photo-induced band to triplet-triplet excited state transitions peculiar for each polyynic chain length considered. Further investigations will pave the way to a complete understanding of the ultrafast processes occurring in exotic organic compounds like polyynes, carbyynes and cumulenes.

\section{References}

1. M. M. Yildizhan, D. Fazzi, A. Milani, L. Brambilla, M. Del Zoppo, W. A. Chalifoux, R. R. Tykwinski, G. Zerbi, J. Chem. Phys. 134, 124512 (2011).

2. C. S. Casari, A. Li Bassi, L. Ravagnan, F. Siviero, C. Lenardi, P. Piseri, G. Bongiorno, C. E. Bottani, P. Milani, Phys. Rev. B 69, 075422 (2004)

3. Y. Zhang, Y. Su, L. Wang, E. S. Kong, X. Chen, Y. Zhang, Nanoscale Res Lett. 6, 577 (2011)

4. F. Cataldo, L. Ravagnan, E. Cinquanta, I. E. Castelli, N. Manini, G. Onida, P. Milani, J. Phys. Chem. B 114, 14834 (2010). 